Saudi Journal of Oral and Dental Research

Abbreviated Key Title: Saudi J Oral Dent Res ISSN 2518-1300 (Print) |ISSN 2518-1297 (Online)

Scholars Middle East Publishers, Dubai, United Arab Emirates

Journal homepage: https://saudijournals.com/sjodr

\title{
Oral Tuberculosis - A common disease at an uncommon site - A Case Report
}

\author{
Dr. Mayank Kumar ${ }^{1}$, Dr. Poonam Elhence ${ }^{2 *}$, Dr. Darwin Kaushal ${ }^{3}$, Dr. Amber Kesarwani ${ }^{4}$ \\ ${ }^{1}$ Assistant Professor, Department of Pathology, Autonomous State Medical College, Ayodhya, India \\ ${ }^{2}$ Professor, Department of Pathology, All India Institute of Medical Sciences, Jodhpur, India \\ ${ }^{3}$ Associate Professor, Department of Otorhinolaryngology, All India Institute of Medical Sciences, Jodhpur, India \\ ${ }^{4}$ Consultant, Department of Otorhinolaryngology, Sanjay Gandhi Hospital, Amethi, India
}

\begin{tabular}{ll}
\hline DOI: $10.36348 /$ sjodr.2021.v06i01.006 & | Received: 26.12.2020 | Accepted: 06.01.2021 | Published: 09.01 .2021 \\
*Corresponding author: Dr. Poonam Elhence &
\end{tabular}

\section{Abstract}

The emerging global resistance to anti-tubercular drugs, and the underdiagnosis of extrapulmonary tuberculosis lesions, warrants an increased awareness of the involvement of Mycobacterium tuberculosis at rare sites of occurrence, such as the oral cavity. The involvement of the tongue, the most common site of oral tuberculosis, can either be primary or secondary. We report a rare presentation of oral tuberculosis in a 45-year-old male who presented with ulcerative lesions on the tongue and epiglottis. Histopathological examination showed chronic granulomatous inflammation with positive Ziehl Neelsen stain. The patient responded well to anti-tubercular treatment. Tuberculosis should be kept as a differential diagnosis of oral cavity lesions so that prompt diagnosis can be offered, leading to better patient management. Efficient treatment in such cases is beneficial for the community, as the patient is a potential source of transmission of tuberculosis.

Keywords: Extrapulmonary lesion, Mycobacterium, oral cavity, tongue, tuberculosis, Ziehl Neelsen stain.

Copyright (C) 2021 The Author(s): This is an open-access article distributed under the terms of the Creative Commons Attribution 4.0 International License (CC BY-NC 4.0) which permits unrestricted use, distribution, and reproduction in any medium for non-commercial use provided the original author and source are credited.

\section{INTRODUCTION}

Tuberculosis, a chronic infectious disease caused by Mycobacterium tuberculosis, mostly affects the lungs. Occurrence of tuberculosis in other organs, termed extra-pulmonary tuberculosis, is seen in $25 \%$ of the cases with only $10 \%-35 \%$ of these cases involving the head and neck region [1]. Tuberculosis of the tongue can be either primary or secondary and is a rare phenomenon. We present a case of tuberculosis of the oral cavity, involving the tongue and epiglottis.

\section{CASE REPORT}

A 45-year-old male presented with complaint of change in voice over a period of fourteen months along with a non-healing ulcer on the left lateral border of the tongue since four months. The ulcer was painful to touch. He gave history of smoking 1-2 cigarettes per day for the past ten years. There was no history of fever, cough, weight loss, dysphagia, haemoptysis, or history of tuberculosis in family members.

On examination, a $1 \times 1 \mathrm{~cm}$ non-indurated ulcerative lesion was present over the anterior part of the left lateral border of the tongue. The tongue mobility was normal. Also, an ulcerative lesion was present over the epiglottis (Figure-1). Remaining part of the endolarynx was congested. No cervical lymph nodes were palpable.

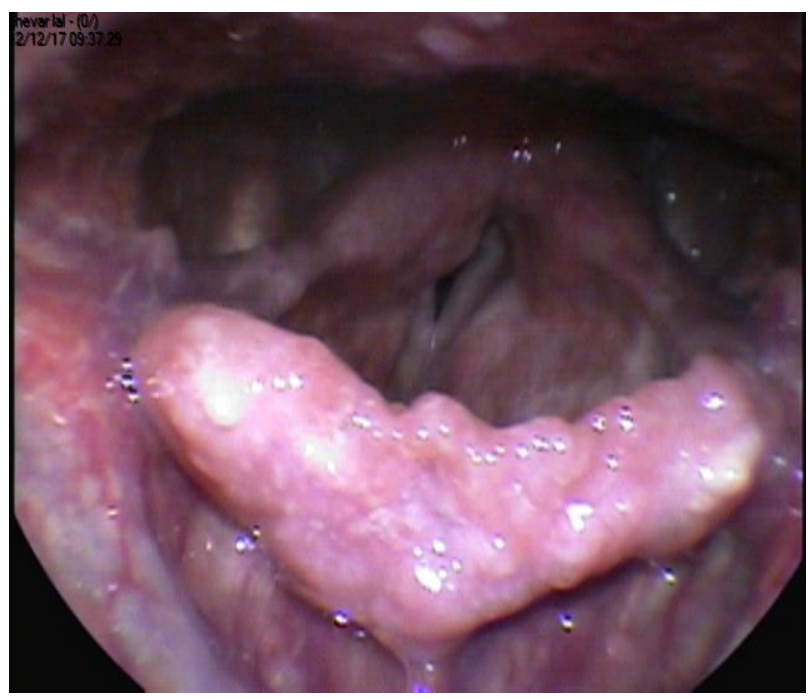

Fig-1: Endoscopic image showing ulcerative lesions on the epiglottis 
Mayank Kumar et al; Saudi J Oral Dent Res, Jan 2021; 6(1): 34-38

A routine complete blood count was within normal limits. Computed tomography (CT) scan of neck and thorax was performed. It revealed enhancement of the epiglottis along with presence of irregular margins and obliteration of vallecula on both sides. Irregular nodules were seen in both the lungs, with some of them showing cavitation and surrounding ground glass opacities. Changes consistent with old lesions of tuberculosis were noted in upper lobes of both the lungs.

Biopsies were taken from both the ulcerative lesions, and submitted for histopathological examination. Tissue from lesion on the tongue showed numerous well-formed discrete epithelioid cell granulomas in the subepithelium, comprising of many Langhans type of giant cells and a peripheral collar of lymphocytes (Figures 2 and 3). Ziehl Neelsen stain to demonstrate acid-fast bacilli was positive (Figure-4). Tissue from lesion on the epiglottis showed an occasional well-defined granuloma along with inflamed granulation tissue and moderate chronic mononuclear cell infiltrate (Figure-5). A diagnosis of chronic granulomatous inflammation consistent with tubercular etiology was offered.

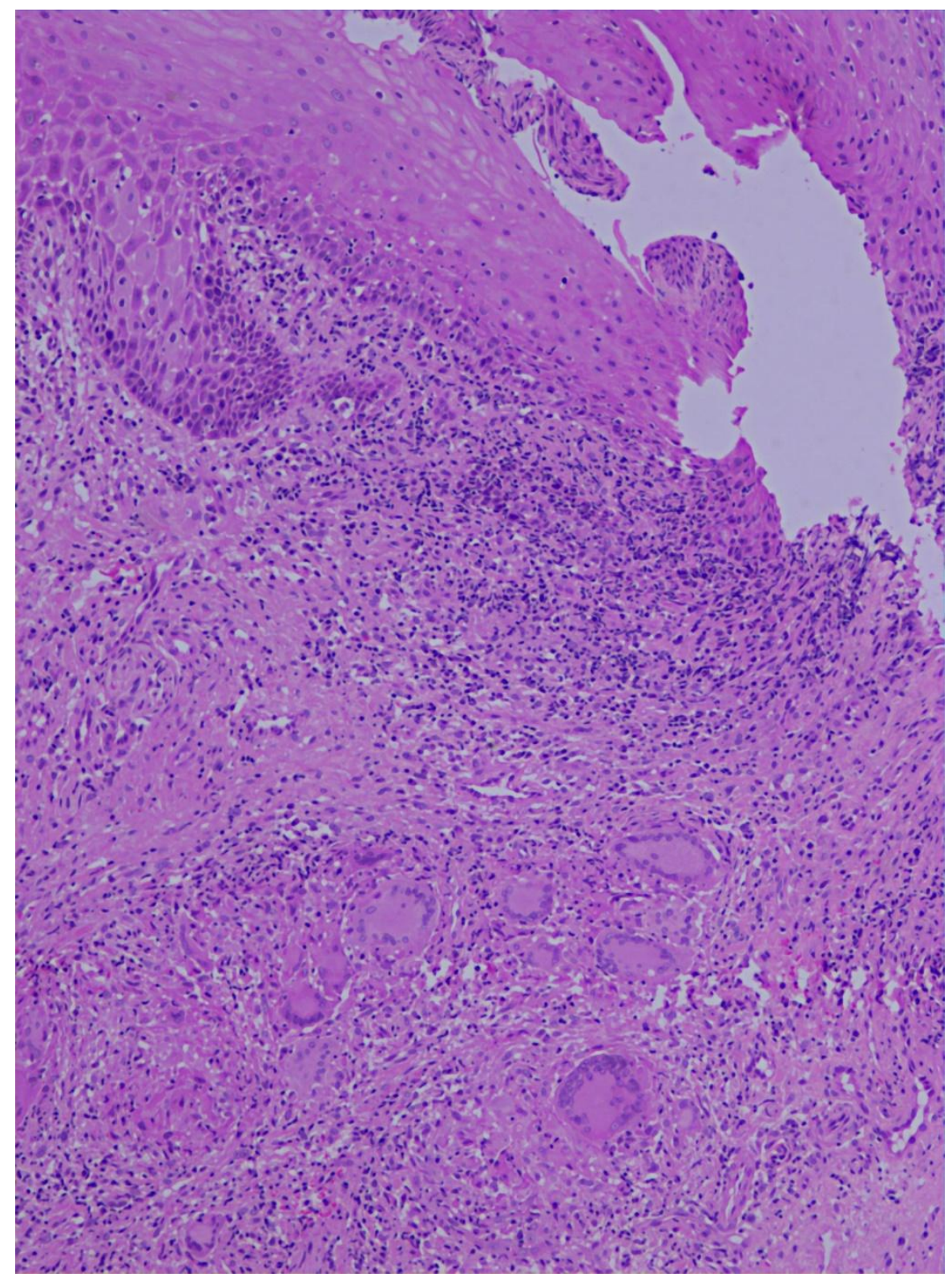

Fig-2: Photomicrograph showing presence of well-formed granulomas with Langhans' type of giant cells below the stratified squamous epithelium lining of tongue (100x) 
Mayank Kumar et al; Saudi J Oral Dent Res, Jan 2021; 6(1): 34-38

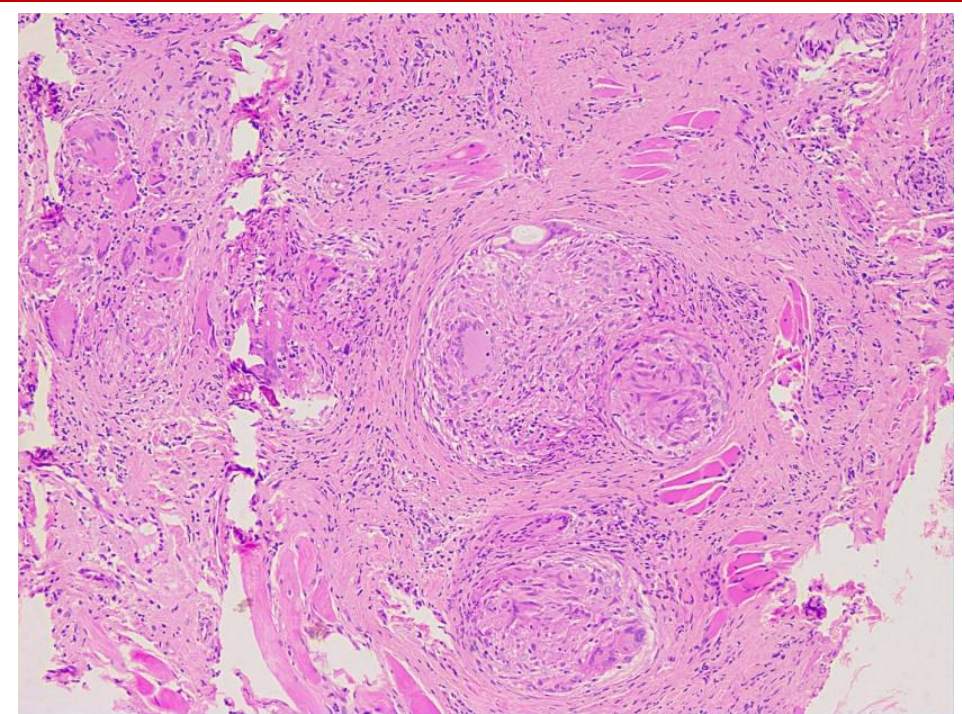

Fig-3: Photomicrograph showing well-formed granulomas with Langhans' type of giant cells in between the skeletal muscle fibres of tongue (100x)

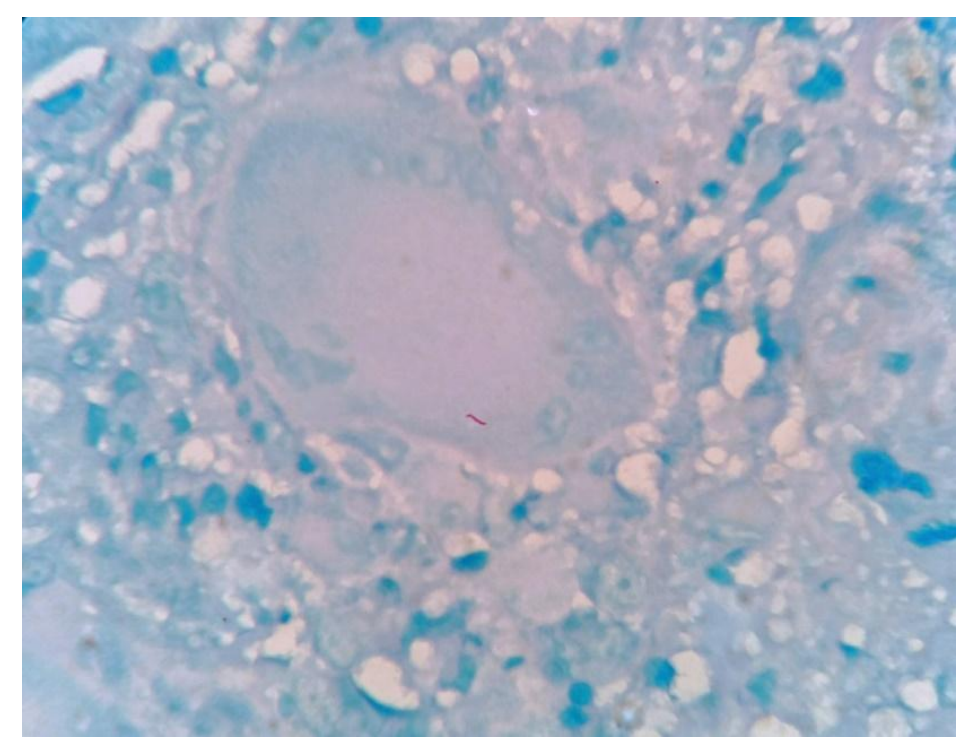

Fig-4: Photomicrograph showing acid fast positive organism on Ziehl Neelsen stain in the tongue (1000x)

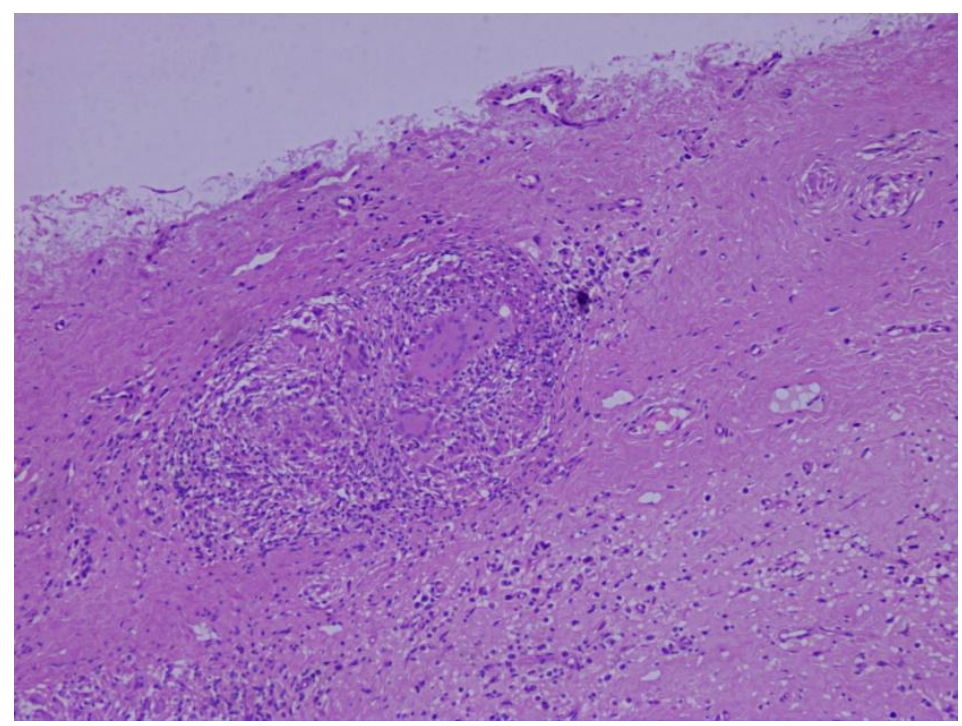

Fig-5: Photomicrograph showing well-formed granuloma in the epiglottis (100x) 
Mayank Kumar et al; Saudi J Oral Dent Res, Jan 2021; 6(1): 34-38

The patient was started on anti-tubercular treatment (ATT). Informed written consent was obtained from the patient, as per institutional guidelines.

\section{DISCUSSION}

As per the estimations of global burden of tuberculosis by the World Health Organization (WHO), one third of the world's population or around two billion people are infected by tuberculosis. The global incidence of tuberculosis is increasing at rate of $1 \%$ per year [2]. However, the tuberculosis of the oral cavity is a rare entity, accounting for only 0.2 to $1.5 \%$ of the cases of extrapulmonary tuberculosis [3]. It is due to this low occurrence rate, that oral tuberculosis is rarely included in the differential diagnosis of lesions in the oral cavity.

The rarity of oral tuberculosis can be attributed to the resistance offered by the stratified squamous epithelium of the oral cavity to colonization by Mycobacterium tuberculosis. This is due to protective mechanisms like thickness of the epithelial layer, cleansing action of saliva and its enzymes, secreted antibodies, and the presence of oral commensal organisms [4]. In addition, the tongue does not contain any significant lymphoid tissue for which Mycobacterium tuberculosis has great affinity [5].

Oral tuberculosis may be primary or secondary. The primary form, which is rarer, is seen more commonly in young individuals and occurs due to direct inoculation of the oral cavity [1]. It is usually seen after a predisposing breach in the oral mucosa as a result of poor oral hygiene, chronic tobacco use, trauma due to irregular tooth, or following tooth extractions [6].

The secondary form, more common in elderly individuals and those with immunocompromised states, occurs due hematogenous or lymphatic spread from the pulmonary site, or by self-inoculation with infected sputum [1]. Sputum formation in pulmonary tuberculosis leads to an increase in concentration of Mycobacterium tuberculosis in the mouth [6].

In both primary and secondary forms of oral tuberculosis, the protective mechanisms are compromised or overwhelmed in some manner.

In the oral cavity, tongue is the most common site of involvement. Other sites include gingiva, palate, floor of the mouth, lips and buccal mucosa [5]. Five pathological types of this lesion are described in the tongue. These include tubercular ulcers, tuberculomas, tuberculous fissures, tubercular papillomas, and tubercular cold abscesses [7]. The commonest among these is an ulcer, characterized by irregular edges and induration [4]. Rarely, the tuberculoma may be so large in size so as to present as hemi macroglossia [6].
The oral lesions in the present case most probably represent secondary form of the disease, as imaging studies suggest presence of old lesions of tuberculosis in upper lobes of both the lungs. The lesions were ulcerative, which is the most common type of lesion in the oral cavity. However, the involvement of the epiglottis, apart from the tongue is a unique finding as it is rarely reported in literature.

The differential diagnosis for ulcers in the oral cavity includes many entities, ranging from malignancy to benign conditions like syphilis, actinomycosis, histoplasmosis, traumatic lesions, recurrent aphthous stomatitis, pemphigoid, erosive form of lichen planus, Crohn's disease, and sarcoidosis [8].

The investigations that can be carried out include exfoliative cytology [9], fine needle aspiration (FNA) or incisional biopsy, depending on the type of lesion.

Prompt diagnosis of oral tuberculosis is necessary for appropriate management of the patient as the condition responds well to anti-tubercular therapy. Also, it is important for the community as a whole since the patient is a potential source of transmission of tuberculosis [1]. Primary care physicians are usually the first point of contact for the general population with the health care system. They should be aware of such rare presentations of common entities, as they play an important role in early diagnosis and initiation of correct treatment.

\section{CONCLUSION}

Tuberculosis should be kept as a differential diagnosis of oral cavity lesions, irrespective of presence or absence of pulmonary disease. Detailed history and examination along with a strong clinical suspicion are necessary to make a diagnosis of oral tuberculosis, which has to be subsequently confirmed by histopathology. The role of primary care physicians in prompt management is very important.

\section{REFERENCES}

1. De Souza, B. C., de Lemos, V. M. A., \& Munerato, C. M. (2016). Oral manifestation of tuberculosis: A case-report. Brazilian Journal of Infectious Diseases, 20(2), 210-213.

2. World Health Organization. (2005). Tuberculosis The Global Burden. Geneva: WHO.

3. Sareen, D., Sethi, A., Agarwal, A. K. (2006). Primary tuberculosis of the tongue: A rare nodular presentation. British Dental Journal, 200(6), 321322.

4. Mohanapriya, T., Singh, K. B., Arulappan, T., \& Dhanasekar, T. (2011). Lingual Tuberculosis. Indian Journal of Tuberculosis, 3(1), 39-41.

5. Al-Rikabi, A. C., \& Arafah, M. A. R. (2011). Tuberculosis of the Tongue Clinically 
Mayank Kumar et al; Saudi J Oral Dent Res, Jan 2021; 6(1): 34-38

Masquerading as a Neoplasm: A Case Report and Literature Review. Oman Medical Journal, 26(4), 267-268.

6. Yadav, S. P. S., Agrawal, A., Gulia, J. S., Singh, S., \& Gupta, A. (2012). Tuberculoma of the Tongue Presenting as Hemimacroglossia. Case Reports in Medicine, 2012(1), 1-3.

7. Khatri, B. K., Jhalla, G. S. (1971). Tuberculosis of tongue: A Case Report. Indian Journal of Tuberculosis, 18(2), 58-59.
8. Krawiecka, E., \& Szponar, E. (2015). Tuberculosis of the oral cavity: An uncommon but still a live issue. Advances in Dermatology and Allergology, 32(4), 302-306.

9. Besra, K., Pathy, P. C., Samantaray, S., \& Rout, N. (2017). Oral tuberculosis diagnosed from exfoliative cytology - two case reports. International Journal of Medical Science and Public Health, 6(2), 432-435. 